\title{
Einstein's invention of Brownian motion
}

\author{
Jürgen Renn* \\ Max-Planck-Institut für Wissenschaftsgeschichte, Wilhelmstr. 44, 10117 Berlin, Germany \\ Published online 14 February 2005
}

Key words Brownian motion, kinetic theory of heat, statistical physics, stochastic processes, thermodynamics of solutions

PACS 05.40.Jc, 05.20.Dd, 05.40.-a, 82.60.Lf

\section{The atomistic revolution ${ }^{1}$}

The impact of Einstein's work

Einstein's 1905 paper on Brownian motion was an essential contribution to the foundation of modern atomism [20]. Atomism as understood in science today presupposes, like its predecessor rooted in the theories of nature from Greek antiquity and from early modern times, that matter is constituted by small entities. But it no longer assumes that the properties and the behavior of these entities can simply be inferred from the familiar physical laws governing our macroscopic environment, nor that a description of matter in terms of its atomistic constituents can be exhaustive. Einstein succeeded in interpreting the irregular movements of small particles suspended in a liquid as visible evidence for the molecular motions constituting the heat of a ponderable body according to the kinetic theory of heat. But he did so by radically changing the understanding of these irregular motions which he no longer conceived as being characterized by a velocity in the classical sense but as a stochastic process that can only be described with the help of statistical methods. It is therefore not surprising that Einstein's work on Brownian motion also became one of the pillars of modern statistical thermodynamics and, more generally, of the physics of stochastic processes.

In the sequel to his groundbreaking work, Einstein published several other related articles, extending the subject to Brownian motion in condensers and the fluctuations of heat radiation. His work aroused widespread interest among physicists and chemists, as indicated by Einstein's correspondence with other scientists interested in the subject, in particular Conrad Röntgen, Richard Lorenz, Marian von Smoluchowski, and The Svedberg. ${ }^{2}$ In 1906 the Polish physicist von Smoluchowski submitted a paper on the kinetic theory of Brownian motion to the Annalen that was stimulated by Einstein's papers but represented results which he had derived independently. While Smoluchowski's argument was different from Einstein's, his results were - apart from a numerical factor - essentially equivalent. Einstein's interpretation of Brownian motion soon also received striking experimental confirmation by Jean Perrin and others. This success furthered the general acceptance of atomism and helped to convert the then still numerous skeptics. Indeed, while in the nineteenth century atomism was widely employed as a working hypothesis in numerous fields of physics and chemistry, it was accepted as a physical reality only after the impressive accumulation

* E-mail: renn@mpiwg-berlin.mpg.de

1 This essay is based on my earlier contributions to the subject, in particular, the editorial note on Brownian motion in [10, 206222], and [44].

2 See Wilhelm Röntgen to Einstein, 18 September 1906 [11, Doc.40]; Richard Lorenz to Einstein, 15 November 1907 [11, Doc.65]; and Einstein to Marian von Smoluchowski, 11 June 1908 [11, Doc. 105]. For evidence of early correspondence between Svedberg and Einstein, see The Svedberg to Einstein, 8 December 1919 [12, Doc. 202]. 
of evidence in the early twentieth century to which Einstein's interpretation of Brownian motion was a key contribution.

This must also have been Einstein's own view. He made a point of sending at least one of his papers on Brownian motion to Ernst Mach, one of the skeptics with regard to atomism, emphasizing the direct relationship between Brownian motion and "thermal motion". ${ }^{3}$ In a popular account from 1915, he wrote: "Under the microscope one, to some extent, immediately sees a part of thermal energy in the form of mechanical energy of moving particles." "In his "Autobiographical Notes", written towards the end of his life, Einstein summarized his view of the influence his work had on Brownian motion [27, p.49]:

The agreement of these considerations [on Brownian motion] with experience together with Planck's determination of the true molecular size from the law of radiation (for high temperatures) convinced the sceptics, who were quite numerous at that time (Ostwald, Mach) of the reality of atoms.

\section{Einstein's work as a historical puzzle}

When turning to the origins of Einstein's eminently successful work on Brownian motion, one is confronted with a puzzle. He did not mention Brownian motion in the title of his paper, which he evidently wrote "without knowing that observations concerning Brownian motion were already long familiar". ${ }^{5}$ In other words, Einstein must have somehow "invented" Brownian motion all by himself. Although he had evidently heard about it, he had no concise empirical information and essentially derived the properties of Brownian motion solely from theoretical considerations. That Einstein was only vaguely familiar with observations of Brownian motion is also suggested in a letter he wrote in May 1905 to his friend and discussion partner Conrad Habicht, a famous letter in which Einstein listed four of the five pathbreaking papers on which he was working during his miracle year. ${ }^{6}$ The paper on Brownian motion was, after the paper on the light quantum and the dissertation on the determination of molecular dimensions, the third on Einstein's list, before the relativity paper, which he had only outlined at that time:

The third proves that, on the assumption of the molecular theory of heat, bodies on the order of magnitude $1 / 1000 \mathrm{~mm}$, suspended in liquids, must already perform an observable random motion that is produced by thermal motion; in fact, physiologist have observed < unexplained $>$ motions of suspended small, inanimate, bodies, which motions they designate as "Brownian molecular motion."

The puzzle of the origin of Einstein's Brownian motion paper raises a number of questions that the following shall attempt to answer: How could Einstein predict the non-classical properties of a phenomenon about which he had apparently no precise information? How was his study of Brownian motion related to his other concerns in 1905, which ranged from the constitution of radiation to the electrodynamics of moving bodies? And why was an explanation of Brownian motion as being due to the motion of atoms and molecules only achieved at the beginning of the twentieth century although both the atomistic hypothesis and the phenomenon itself were long familiar at that time? We shall begin our discussion by addressing the last question.

3 Einstein to Ernst Mach, 9 August 1909 [11, Doc. 174] and 17 August 1909 [11, Doc. 175]

4 [26, p. 261].

5 [27, p. 44; translation, p. 45].) See also Einstein to Michele Besso, 6 January 1948 [1, Call Nr. 7-382.00], and Einstein to Carl Seelig, 15 September 1952 [1, Call Nr. 39-040].

6 Einstein to Conrad Habicht, 18 or 19 May 1905 [11, Doc. 27].

7 "Die dritte beweist, daß unter Voraussetzung der molekularen Theorie der Wärme in Flüssigkeiten suspendirte Körper von der Größenordnung 1/1000 $\mathrm{mm}$ bereits eine wahrnehmbare ungeordnete Bewegung ausführen müssen, welche durch die Wärmebewegung erzeugt ist; es sind < unerklärte $>$ Bewegungen lebloser kleiner suspendirter Körper in der That beobachtet worden von den Physiologen, welche Bewegungen von ihnen "Brownsche Molekularbewegung" genannt wird." Unless indicated otherwise, translations are taken from the English companion volumes to the "Collected Papers of Albert Einstein." 


\title{
A marginal problem of classical science
}

\author{
The exclusion of alternative explanations
}

The first systematic study of the irregular movement of microscopic particles suspended in a liquid goes back to the botanist Robert Brown, who published his careful observations in $1828 .{ }^{8}$ He examined a great variety of materials which he suspended in a liquid. These ranged from the pollen of plants to fragments of an Egyptian sphinx. He also explored an equally great variety of possible causes of the irregular motion of the suspended particles, ranging from currents within the liquid via an interaction among the particles to the creation of small air bubbles. In this way Brown was able to exclude a number of potential explanations of the irregular movement of the suspended particles and, in particular, the claim that this is a characteristic of organic materials only and somehow an expression of "life." Nevertheless, Brownian motion failed to become a subject of broad interest to physicists, at least until the middle of the nineteenth century. Meanwhile several papers appeared in which the influence of specific circumstances on the properties of Brownian motion were studied, such as the temperature of the liquid, capillarity, convection currents of the liquid, evaporation, the illumination of the particles, electrical forces, and the role of the environment. ${ }^{9}$

While from today's perspective, these early works may appear to be more or less fruitless and part of a prehistory that may just be disregarded, the knowledge accumulated by these works turned out to be crucial for the later identification of Brownian motion as a special case of the kind of motion that we call heat. First, all the various experiments taken together pointed to the persistence and the ubiquity of the phenomenon. Second, they increasingly excluded a priori possible explanations that made use of the specific circumstances under which the phenomenon was produced. If the explanations by Einstein and von Smoluchowski found such a rapid and successful acceptance as evidence in favor of an atomistic constitution of matter, this was not least due to the fact that alternative explanations of Brownian motion had been thoroughly pursued over nearly a century and eventually discarded after careful examination.

\section{Brownian motion as a challenge for the kinetic theory}

Since the mid-nineteenth century, several authors considered the kinetic theory of heat as a possible explanation of Brownian motion. ${ }^{10}$ This is hardly surprising as the kinetic theory, as developed by Rudolf Clausius, James Clerk Maxwell, Ludwig Boltzmann and others, became an ever more powerful tool to explain thermal phenomena on a mechanical basis. Still there was a variety of factors that could be taken into account when attempting to explain Brownian motion within this framework, such as the temperature and viscosity of the liquid, the differences in specific heats of the particles and the liquid, as well as the magnitude and velocity of the particles. In addition, the role of additional factors outside the scope of the kinetic theory, such as electric interactions, could still not be excluded and gave rise to further investigations. But the potential complexity of the phenomenon was not the only reason it remained in a kind of "epistemic isolation," that is, why it failed to become a key subject of the great works on the kinetic theory of heat.

One of the reasons for the marginal role that Brownian motion continued to play in nineteenth-century physics was a matter of perspective. Indeed, the focus of the kinetic theory was oriented more towards a reconstruction of the laws of phenomenological thermodynamics than towards the discovery of deviations from these laws, even if these were the statistical fluctuations that must occur if the interpretation of heat as a kind of motion is correct. ${ }^{11}$ Boltzmann's Gastheorie, for instance, explicitly denies that the thermal motion of molecules in a gas leads to observable motions of suspended bodies. ${ }^{12}$ Another reason was the intrinsic difficulties of applying the kinetic theory to Brownian motion. Since the 1870s, several scientists had pursued the idea that Brownian motion might be explained as the result of collisions between suspended

\footnotetext{
8 See [4].

9 For contemporary reviews of research on Brownian motion, see [50] and [13]. For historical accounts, see [5,36].

${ }^{10}$ For historical discussion, see [5, §3].

11 See the historical discussion in [44].

12 See [3, pp. 111-112].
} 
particles and the molecules of the liquid, among them Delsaulx, Carbonelle, and Gouy. ${ }^{13}$ Gouy supported this explanation by performing further experiments excluding alternative accounts. While the qualitative explanation of Brownian motion with the help of the kinetic theory thus became ever more plausible, serious problems occurred as soon as such an explanation made use of quantitative arguments.

It was this kind of quantitative argument that was used by the cytologist Karl von Nägeli in 1879 against the kinetic explanation of Brownian motion. ${ }^{14}$ The argument was based on the equipartition theorem at the center of the kinetic theory. According to this theorem, in thermal equilibrium the energy of a physical system is equally distributed over its internal degrees of freedom, the energy portion of each single degree of freedom being proportional to the absolute temperature. It was therefore possible to calculate the average velocity of the molecules of the liquid, and then use the laws of elastic collision to obtain the velocity of a suspended particle. Nägeli concluded from this argument that the velocity of such a particle, because of its comparatively large mass, would be vanishingly small. This internal contradiction of an explanation of Brownian motion as the motion of a very large molecule in thermal equilibrium with all the smaller molecules of the liquid was confirmed in 1900 by the work of Felix Exner, actually a supporter of the kinetic explanation. ${ }^{15}$ He performed extensive measurements of the velocity of Brownian motion and observed that it decreases when larger particles are suspended and increases with rising temperature, as must be the case according to the kinetic theory. When he calculated, however, the kinetic energy of the molecules on the basis of his velocity measurements, he found values that were dramatically smaller than those implied by the kinetic theory of heat.

Consequently, by the turn of the century, Brownian motion had emerged as a veritable challenge to classical physics, even if this challenge was not broadly acknowledged due to the apparent marginality of the phenomenon, at least from the perspective of the majority of the physics community. With practically all other accounts excluded, the kinetic theory had emerged as the most viable option for explaining the phenomenon, yet failed to provide an adequate quantitative understanding.

\section{Preparing for a breakthrough}

\section{Einstein's perspective}

How did Einstein encounter the problem of Brownian motion and why was he able to provide a solution to this problem that had apparently escaped his predecessors? Einstein's perspective distinguished itself from that of the majority of his contemporaries by his broad orientation beyond the narrow confines of subdisciplinary specialization. Since his early student days, he was interested in atomism as a conceptual tool for identifying hidden links between physical phenomena, which otherwise seemed to have no connection, such as the specific heats of solids and their optical transparency, or the thermal and electrical conductivity of metals. He also speculated about the relation between molecular and gravitational forces and about the possibility of a direct conversion of the kinetic energy of molecules and atoms into light. The search for a conceptual unity of physics, which became the hallmark of Einstein's research, had probably been prepared by his early reading of popular scientific literature in which the heritage of the Romantic idea that science could account for the unity of nature was preserved. ${ }^{16}$

Atomismus was, however, not only one of the principal conceptual tools employed in Einstein's quest for a scientific world view, but was also widely exploited in contemporary professional research within the disciplines and subdisciplines of classical science, which ranged from Lorentz's electron theory of electromagnetism via Boltzmann's kinetic gas theory to chemistry. In view of this spread of atomistic ideas, it is rather surprising that attempts to relate the various usages of the concept to each other, in order to extract a coherent overall picture, were not more prominent. Attempts at a conceptual synthesis had been

\footnotetext{
13 See the discussion in [13].

14 See [34].

15 See [30]

${ }^{16}$ For a more extensive discussion, see [44].
} 
almost obligatory for any scientist in the age of the Scientific Revolution. But in the nineteenth century, the age of specialization, these attempts were no longer part of the ordinary pursuit of science and were rather left to a few philosopher-scientists such as Ernst Mach and Henri Poincaré, whose works the young Einstein ardently devoured. He read, for instance, Poincaré's Science et hypothèse, which contains a brief discussion of Gouy's work on Brownian motion, and emphasizes Gouy's argument that Brownian motion violates the second law of thermodynamics, i.e,. the principle of the irreversibility of thermodynamic processes. ${ }^{17}$ Einstein was therefore not only familiar with the potential of atomism as a conceptual bond between phenomena studied in isolation from each other because of the specialist outlook of contemporary science. He was also aware of the precarious status of concepts such as atoms and the ether, which were often uncritically presupposed in contemporary scientific arguments, without carefully examining their meaning and their relation to empirical evidence that was not just limited to the special problem on which a particular investigation happened to focus.

During Einstein's student years, the kinetic theory of heat was the subject of a heated controversy between Ernst Mach, Wilhelm Ostwald, Georg Helm, and Ludwig Boltzmann. ${ }^{18}$ Mach rejected the existence of entities not directly accessible to sense-experience, and was skeptical, in particular, about the existence of atoms. Although Einstein criticized Boltzmann for a lack of emphasis on the comparison of his theory with observation, ${ }^{19}$ he enthusiastically embraced the atomistic principles of Boltzmann's theory. ${ }^{20} \mathrm{He}$ must have therefore found it challenging when he read in Boltzmann's Gastheorie ${ }^{21}$, that Boltzmann, presumably reacting to the above-mentioned controversy, suggested that he was isolated in his support of the kinetic theory. ${ }^{22}$ Indeed Einstein's interests soon turned from the details of atomistic explanations to the quest for facts, "which would guarantee as much as possible the existence of atoms of definite finite size," as he later remembered. ${ }^{23}$

The theory of solutions

There can be little doubt, however, that Einstein's perspective on the problem of Brownian motion was as much shaped by the specific problems he dealt with in his prior research as by the general, philosophical outlook outlined above. Even in his first two papers, published in 1901 and 1902 and later disqualified as worthless beginner's papers, ${ }^{24}$ he familiarized himself with some of the ideas that figured in his later work on Brownian motion, in particular the nature of diffusion processes and the application of thermodynamics to the theory of solutions. ${ }^{25}$ In [17], for instance, he suggested replacing semipermeable walls in thermodynamic arguments with external conservative forces, a method he stated to be particularly useful for treating arbitrary mixtures. In his subsequent papers on statistical physics, Einstein generalized the idea of external conservative forces, ${ }^{26}$ and, as we shall see in more detail below, noted the significant role of fluctuations in statistical physics.

\footnotetext{
${ }^{17}$ See [43, p. 209].

${ }^{18}$ For Einstein's reading of Mach, see Einstein to Mileva Marić, 10 September 1899 [9, Doc. 54]; for his reading of [37, 1893], see Einstein to Wilhelm Ostwald, 19 March 1901 [9, Doc. 92]; for his reading of [2,3], see Einstein to Marić, 10 September 1899, 13 September 1900, and 19 September 1900 [9, Docs. 54, 75, and 76].

${ }^{19}$ On 30 April 1901, Einstein wrote to Mileva Marić: "At present I am again studying Boltzmann's theory of gases. Everything is very nice, but there is too little stress on the comparison with reality" ("Ich studiere gegenwärtig wieder Boltzmanns Gastheorie. Alles ist sehr schön, aber zu wenig Wert gelegt auf den Vergleich mit der Wirklichkeit.") [9, Doc. 102].

${ }^{20}$ Einstein to Mileva Marić, 13 September 1900 [9, Doc. 75].

${ }^{21}$ See the preceding note.

${ }^{22}$ See the preface to [3]; for accounts of the dispute, see [6, pp.96-98;], [14, pp.416ff.]

${ }^{23}$ See [27, p. 44; translation, p. 45].

${ }^{24}$ See $[16,17]$. For their qualification, see Einstein to Johannes Stark, 7 December 1907 [11, Doc. 66]. See also the editorial note "Einstein on the Nature of Molecular Forces" in [10, pp.3-8].

${ }^{25}$ For a discussion of Einstein's earlier interest in diffusion, see the editorial note "Einstein's Dissertation on the Determination of Molecular Dimensions” in [10, 177-179].

${ }^{26}$ See, in particular, $[18, \S 10]$.
} 
Einstein's contemporary correspondence suggests that the theory of solutions, including the issues of semipermeable membranes and osmotic pressure, must have played an even larger role in his thinking than is directly apparent from his published papers. In 1903, he discussed the concepts of semipermeable membrane and osmotic pressure in his correspondence with Michele Besso, and expressed interest in Sutherland's hypothesis on the mechanism of semipermeable membranes. ${ }^{27}$ The theory of solutions provided, as elaborated by Jacobus H. van't Hoff and later by Walther Nernst, a concise analogue to the kinetic theory of gases, and therefore offered an important field of exploration to someone like Einstein, who was interested in extending the range of the applicability of atomistic ideas. ${ }^{28}$ The theory of solutions must also have been central to the thesis Einstein submitted for his doctoral degree, a thesis he eventually withdrew. ${ }^{29}$ He used this again in 1905 as the central subject of the dissertation with which he finally succeeded in obtaining his degree.

Einstein's doctoral thesis provided much of the framework essential to his analysis of Brownian motion. ${ }^{30}$ It was also motivated by a goal similar to the one in his paper on Brownian motion, i.e., to offer evidence for the existence of atoms and molecules and to determine their size. The dissertation proposed a new method for measuring atomic dimensions, explaining how Avogadro's number could be found by considering large sugar molecules in solution. The basic procedure was to set up two equations for two unknowns, from which Avogadro's number and the size of the solute molecules could then be calculated. Physically, one of the equations was derived from the change in viscosity due to the addition of sugar molecules to the solution, while the other used a relation between the diffusion coefficient of the sugar molecules and the viscosity of the solution. The first of these equations was derived from rather involved hydrodynamical calculations. The other equation, relating diffusion and viscosity, turned out to be crucial for the analysis of Brownian motion as well. It may therefore be worthwhile to examine its conceptual roots more closely. Its derivation is based ultimately on establishing a bridge between a bulk phenomenon, diffusion, and the motion of an individual particle as affected by viscosity acting as a friction force due to the environment of the particle. How did Einstein manage to build this bridge and how did the very idea to look for such a relation emerge?

\section{The relation between diffusion and viscosity}

These questions lead back to Einstein's first two papers, which deal with the theory of solutions and to his continued concern with their thermodynamic properties. In his second paper, dedicated to the properties of electrolytic solutions, he questioned the legitimacy of applying the laws of thermodynamics and concepts such as osmotic pressure to such solutions, even if no semipermeable membranes are available to provide experimental meaning to such pressure. He addressed this question by claiming that such devices can be substituted by conservative forces acting on the substances under consideration. In the derivation of the relation between diffusion and viscosity in his later paper on Brownian motion, he made use of such forces, which now played the role of an intermediate between the motion of individual particles and the bulk process of diffusion.

In this paper, Einstein considered particles suspended in a liquid and analyzed the dynamic equilibrium of these particles under the assumption that the individual particles are subject to the influence of a force depending only on position. This force is hence an example of the kind of fictitious conservative forces that Einstein had introduced earlier to replace unrealizable semipermeable membranes in thermodynamic considerations. It is therefore not surprising that his thermodynamic considerations led him to conclude that the position-dependent force is counterbalanced by a force due to the osmotic pressure. Clearly, this conclusion relates a force acting on the individual particles to a bulk property of the suspended particles -

\footnotetext{
27 See Michele Besso to Einstein, 7-11 February 1903 [11, Doc. 6], which indicates that there was additional correspondence on this subject. See [51] for Sutherland's hypothesis.

28 See [55] and [35].

${ }^{29}$ See the extensive discussion in [44].

30 See the editorial note "Einstein's Dissertation on the Determination of Molecular Dimensions," in [10, pp. 177-179] and [44].
} 
their osmotic pressure. Einstein then considered the dynamic equilibrium of the suspended particles from a second perspective, i.e., as a balance of the motion of the individual particles under the influence of the fictitious force and a process of diffusion. To describe the motion of the particles in the liquid, he relied on Stokes' law between the force exerted, the viscosity of the liquid, and the velocity attained by the particles. To describe the diffusion process, Einstein simply made use of the definition of the diffusion coefficient as relating transport and density of the suspended particles.

On the basis of these results, Einstein was now able to couple the two balance equations, one derived from a thermodynamic argument, and the other from relating diffusion to the motion of the individual particles determined with the help of Stokes' law. He could thus eliminate the fictitious force and directly establish a relation between the diffusion coefficient and osmotic pressure. As the latter involves, according to the kinetic theory of heat, Avogadro's number, Einstein finally arrived at an expression of the diffusion coefficient in terms of atomic sizes.

$$
D=\frac{R T}{N} \frac{1}{6 \pi k P}
$$

where $R$ the gas constant, $T$ the temperature, $N$ Avogadro's number, $k$ the viscosity, and $P$ the radius of the solute molecules or the suspended particles.

In his dissertation, he used this equation together with the equation relating atomic sizes to the change of viscosity derived from hydrodynamic considerations to derive values for the atomic dimensions from experimental data on diffusion and viscosity. In his paper on Brownian motion, he rederived the viscositydiffusion equation, albeit in a more elegant manner. He now made use of the methods of statistical mechanics that he had developed in previous years to describe the irregular motion of suspended particles.

\section{Statistical mechanics and heat radiation}

Einstein's establishment, independently of Willard Gibbs, of statistical mechanics between 1902 and 1904 was motivated by the quest mentioned above to extend the methods of the kinetic theory beyond gases to include a wide range of physical systems such as the electron gas in metals and heat radiation, not least in order to provide additional evidence for the atomic hypothesis. The methods he developed imposed only the most general requirements on the systems studied and did not depend on knowledge of the detailed interaction between the constituents of a systems as is the case in kinetic gas theory, where collision dynamics plays a major role. As Einstein wrote in a letter to his friend Marcel Grossmann in 1904, who at that time was a student of mathematics working on non-Euclidean geometry: ${ }^{31}$

There is a remarkable similarity between us. ... You treat geometry without the parallel axiom, and I treat the atomistic theory of heat without the kinetic hypothesis.

Einstein's generic approach to the statistical properties of physical systems may thus appear to have been well-suited to examine the properties of a phenomenon such as Brownian motion. But by 1904, he had evidently neither heard about it nor could he conceive its existence. In his last paper on statistical mechanics, he did study fluctuation phenomena and even derived an expression for mean square deviations from the average value of the energy of a system, which he interpreted as expressing a condition for the stability of a physical system involving Boltzmann's constant, thus giving a new meaning to this constant. ${ }^{32}$ But when it came to the issue of the observability of such fluctuations, Einstein claimed that radiation in thermal equilibrium was the only system for which experience suggested that it exhibits observable fluctuations. His argument was that, for a radiation cavity whose linear dimensions are chosen so as to be comparable to

31 "Es waltet eine merkwürdige Ähnlichkeit zwischen uns. ... Du behandelst die Geometrie ohne das Parallelenaxiom, ich die atomistische Wärmelehre ohne die kinetische Hypothese.” Einstein to Marcel Grossmann, 6 April 1904 [11, Doc. 17].

32 At this time, however, Einstein regarded black-body radiation as the only physical system for which experience suggests the existence of observable energy fluctuations." See [19, p.361]. 
the wavelength corresponding to the maximum energy in the black body spectrum, the fluctuations should be of the same order of magnitude as the mean energy.

The numerical values given to illustrate this claim indicate that Einstein must had used Wien's formula to describe the energy spectrum of heat radiation. ${ }^{33}$ He may well have considered as early as 1904 what we would call today a photon gas, starting from the speculative assumption that heat radiation may be conceived of as a collection of light quanta whose energy is given by their frequency according to $E=h \nu$. He did, however, then cast his arguments into a form that made them independent from a specific interpretation of heat radiation and only came back to the light quantum hypothesis a year later, now as a means of interpreting Planck's radiation formula, at least in the range in which it can be reasonably approximated by Wien's formula.

\section{Revisiting fluctuations}

By 1905, Einstein's views of the observability of fluctuation phenomena had changed. He had now assembled all the tools necessary to construct a model of observable fluctuation phenomena in a material system by integrating the results achieved whilst writing his dissertation, i.e., the study of dissipation and diffusion phenomena, and those accumulated whilst studying fluctuation phenomena in the context of statistical mechanics and its application to heat radiation. First of all, fluctuations in heat radiation could be related directly to a material process if a mirror is exposed to them, which, as a consequence of the radiation impinging on it and the friction force it suffers at the same type should exhibit Brownian motion-like behavior. This thought experiment was discussed at length in Einstein's later publications, but was apparently already conceived by 1905, as is indicated by later recollections. It represents, so to speak, the missing link between Einstein's principal concerns at the time - heat radiation, statistical physics, and the electrodynamics of moving bodies.

Second, and perhaps of even greater consequence to Einstein's invention of Brownian motion, the argument at the core of his dissertation with which he had inferred that the observability of fluctuations in the case of heat radiation can be transferred directly to the model of large molecules in solution. Increasing the linear dimensions of such particles in analogy to considering wavelengths comparable in size to the radiation cavity would not change the character of the particles as partaking in a world governed by the kinetic theory of heat, but might actually make their random motions visible. The crucial function of the dissertation model was thus to provide a framework in which scaling of this kind, first conceived for the case of radiation, made physical sense for a material process. All that was needed was to mentally transform a solvent with large molecules into a suspension with minuscule but observable particles. Such a transition was particularly plausible as the commonly made distinction between suspensions and solutions in nineteenth-century chemistry had, by the turn of the century, gradually lost its absolute character. ${ }^{34}$ The absence of any fundamental difference between solutions and suspensions became clear in 1902, when observations using the newly invented ultramicroscope ${ }^{35}$ made it possible to resolve many colloidal solutions into their constituents. ${ }^{36}$

In summary, Einstein's invention of Brownian motion was just as much prepared by his quest to identify evidence in favor of the atomic hypothesis as by the specific research problems he had tackled, in particular, in the course of his long-standing interest in the theory of solutions. Combining a model that had assumed a central role in this pursuit - the model of suspended particles undergoing diffusion in a liquid - with his search for observable fluctuation phenomena, he was naturally led to consider the irregular motion that must

\footnotetext{
${ }^{33}$ For this claim, see [46].

${ }^{34}$ For a contemporary discussion of the distinction between solution and suspensions, see the introduction to [56]. For a discussion of colloidal chemistry and its relation to the study of Brownian motion, see [36, pp. 98-102].

35 The ultramicroscope, developed by Henry Siedentopf and Richard A. Zsigmondy, is based on a new illumination technique that makes it possible to observe the diffraction discs of otherwise invisible objects; it increased the limit of visibility to approximately $5 \times 10^{-1}$ micron. For a contemporary discussion of ultramicroscopes, see [8, Chap. 3]

${ }^{36}$ See [49].
} 
be exhibited by such particles. The formula he had derived for expressing the diffusion coefficient in terms of atomic dimensions and the size of the particles would now make it possible to extract information about the atomic scale from the irregular motion of the suspended particles to the atomic scale, if this motion of individual particles could be related to the bulk property of diffusion. To bridge this last gap, Einstein needed a crucial conceptual leap in his analysis of Brownian motion, conceiving it as a kind of process hitherto unknown in classical physics. Before coming to this last step, it is helpful to reconsider the problem of Brownian motion from a somewhat larger perspective, comparing it to the other problems for which Einstein achieved equally important conceptual breakthroughs, in particular, the problem of heat radiation, which triggered the quantum revolution, and the problem of the electrodynamics of moving bodies, which gave rise to the relativity revolution.

\section{Brownian Motion as a Borderline Problem}

The three partite division of classical physics

The physical problems at the center of Einstein's annus mirabilis 1905 have a common feature that becomes evident only when considered from the perspective of the long-term development of physical knowledge. They may be characterized as borderline problems of classical physics because they are all situated at the intersection of two of the three major subdomains of contemporary physics: mechanics, thermodynamics, and electrodynamics. The problem of heat radiation in thermal equilibrium thus constituted a borderline problem between thermodynamics and the theory of radiation, i.e., electrodynamics. The electrodynamics of moving bodies represented a borderline problem between electrodynamics and the theory of motion, i.e., mechanics. And problems of the kinetic theory of heat, such as Brownian motion, can be understood as borderline problems between thermodynamics and mechanics. Borderline problems require the integration of knowledge resources from different domains. They also serve as catalysts that unveil conceptual conflicts between these domains such as the conflict between the constancy of the speed of light and the relativity principle in the case of the electrodynamics of moving bodies.

The hidden bond between Einstein's revolutionary papers of 1905 was their precise concern with such borderline problems. This concern emerged, in Einstein's intellectual biography, in connection with his quest, mentioned above, to establish a conceptual unity of physics by probing the explanatory power of what may be called a speculative or "interdisciplinary" atomism. In this way, the 1905 problems entered his intellectual focus as problems related to more than one conceptual framework of classical physics rather than special issues pertaining to one of its subdomains. Brownian motion could thus be perceived as a borderline problem in the sense that it represented a challenge both for classical phenomenological thermodynamics and its counterpart, the kinetic theory of heat. It constituted a concrete problem allowing the confrontation of their different perspectives. As a consequence, contradictions were engendered that suggested a rethinking and restructuring of the given architecture of knowledge.

\section{Brownian motion as a paradox}

Einstein addressed the problem of Brownian motion in his 1905 paper in exactly this way, as a paradox between thermodynamics and the kinetic theory. He argued that, from the perspective of phenomenological thermodynamics, small particles suspended in a liquid should, after a while, simply achieve a thermal equilibrium with the surrounding liquid and certainly not continue to perform irregular motions. According to classical thermodynamics, the second law is not just a statistical assertion but claims absolute validity. The scepticism toward a merely statistical interpretation of the second law precisely represented a serious obstacle to the conception of Brownian motion as the result of collisions between the suspended particles and the molecules of the liquid.

From the perspective of the kinetic theory, on the other hand, nothing distinguishes these particles in principle from atoms and molecules but their size. They should therefore be exposed to the permanent 
collisions ensuring the thermal equilibrium in the liquid and partake themselves in this thermal motion. But, as mentioned earlier, Brownian motion also confronted the kinetic theory with a serious problem, the lack of agreement between the observed velocities and the velocities calculated theoretically on the basis of the equipartition theorem. It is remarkable that the equipartition theorem, crucial to Einstein's investigations of heat radiation and consolidated in the context of his work on statistical mechanics, plays no role in his work on Brownian motion. He thus may well have been aware of the difficulty of ascribing a velocity in the ordinary sense to the particles suspended in a liquid.

The irregular motion of such particles hence manifests a conflict between two domains of classical physics in a way similar to the conflict between the relativity postulate and the constancy of the speed of light embodied in the electrodynamics of moving bodies, as well as to the conflict between the assumption of a continuum of wavelengths and the assumption of an equipartition of energy in the case of heat radiation. Einstein's reaction to these conflicts was similar in all three cases as well, and distinguished itself in a similar way to that of most of his contemporaries, who looked at such problems from a specialist perspective. While it was plausible to assume that these conflicts were due to a failure somewhere buried in the conceptual foundations of one of the domains involved, Einstein's remarkable overview of the knowledge of classical physics as well as his philosophical acumen caused him to be skeptical with regard to all of these domains and dare to look for new concepts that were capable of overcoming what he saw as a fundamental crisis of classical physics.

Instead of relying on the classical concept of an ether as used in Lorentz's electron theory, abandoning essentials of classical mechanics such as the relativity principle, Einstein audaciously conceived of new concepts of space and time to resolve the problems of the electrodynamics of moving bodies. He was thereby able to preserve insights from both electrodynamics and mechanics, rather than sacrificing one for the sake of the other. In his treatment of the behavior of small suspended particles, Einstein similarly combined insights of the kinetic theory and of thermodynamics, i.e., of micro- and macrophysics, without reducing one to the other. Instead he proposed new laws for the domain of "mesoscopic" physics, which was recognized for the first time as an autonomous level of physical knowledge.

\section{Reinterpreting the results of classical physics}

As we have seen above, Einstein inferred from the kinetic theory that a suspension of small particles must possess an osmotic pressure just as in the case for a solution of molecules. If this pressure is distributed in a spatially inhomogeneous way, it gives rise to a compensatory diffusion process whose bulk properties can be calculated, as also discussed above, with help of Stokes' law determining the moveability of the particles in a viscous fluid. In this way, Einstein obtained an equation for the diffusion coefficient figuring in the partial differential equation, determining the relation between spatial and temporal change of the concentration $f(x, t)$ of a substance in solution:

$$
\frac{\partial f}{\partial t}=D \frac{\partial^{2} f}{\partial x^{2}},
$$

where $D$ is the diffusion coefficient. This equation was first established by Adolf Fick, following the work of Fourier for the conduction of heat and that of Ohm for the conduction of electricity [31]. Einstein now reinterpreted this equation in a way analogous to his reinterpretation of the Lorentz transformations for the electrodynamics of moving bodies and to his reinterpretation of Planck's black-body formula. While largely preserving the technical framework of these results in the works of Lorentz and Planck respectively, Einstein had profoundly changed their conceptual meaning, thus creating the new kinematics of the theory of special relativity and introducing the revolutionary idea of light quanta. He did so in a process of reflection that may be described as a "Copernicus process" since Copernicus as well had largely kept the deductive machinery of traditional astronomy when changing its basic conceptual structure [45]. In the context of his work on fluctuation phenomena, Einstein similarly gave a radically new interpretation to the traditional diffusion equation, thus effectively "inventing" Brownian motion as a theoretical concept. 
Instead of considering the diffusion equation as describing the overall distribution of a solute substance, Einstein now interpreted it as determining the probability distribution of the irregular displacements of the individual particles. The introduction of such a distribution could draw on his previous experience with such probability distributions in his work on statistical mechanics. ${ }^{37}$ In his paper, he wrote with regard to the above equation: ${ }^{38}$

This is the familiar differential equation for diffusion, and $D$ can be recognized as the diffusion coefficient. Another important consideration can be linked to this development. We assumed that all the individual particles are referred to the same coordinate system. However, this is not necessary since the motions of the individual particles are mutually independent. We will now refer the motion of each particle to a coordinate system whose origin coincides at time $t=0$ with the position of the center of gravity of the particle in question, with the difference that $f(x, t) d x$ now denotes the number of particles whose $X$-coordinate has increased between the times $t=0$ and $t=t$ by a quantity lying between $x$ and $x+d x$. Thus, the function $f$ varies according to equation(1) in this case as well.

In this way, Einstein managed to identify the irregular motion of the suspended particles, now described not as a movement in the ordinary sense along a continuous trajectory but as a stochastic process governed by the function $f(x, t)$, as the elementary process corresponding to diffusion as a bulk phenomenon. He assumed the existence of a time interval that was short with respect to the observation time, but long enough to treat the motions of a suspended particle in two successive time intervals independently of each other. The displacement of the suspended particles can then be described by a probability distribution that determines the number of particles displaced by a certain distance in each time interval.

On the basis of this new interpretation, the solution of the diffusion equation, when combined with Einstein's expression for the diffusion coefficient, now results in an expression for the mean square displacement, $\lambda_{x}$ as a function of time. Einstein suggested that this expression could be used experimentally to determine Avogadro's number $N$ :

$$
\lambda_{x}=\sqrt{t} \sqrt{\frac{R T}{N} \frac{1}{3 \pi k P}},
$$

where $t$ is the time, and as before $R$ the gas constant, $T$ the temperature, $k$ the viscosity, and $P$ the radius of the suspended particles.

\section{Repercussions}

Brownian motion continued to play the role of a borderline problem in Einstein's subsequent publications, in which he related it not only to thermodynamics and the kinetic theory, but to electrodynamics and to radiation theory as well. In his second paper on the subject, he elaborated on the relation between Brownian motion and the foundations of the molecular theory of heat [21, p. 371]. There he took up his earlier result for energy fluctuations derived in $1904,{ }^{39}$ and applied it to a system subject to an external force in order to calculate the probability of deviations from the equilibrium value - due to irregular molecular motions of a suitable observable parameter of the system. He also derived a formula for the vertical distribution of

37 For Einstein's first use of probability distributions in his papers on statistical physics, see [18, p. 422].

38 "Dies ist die bekannte Differenzialgleichung der Diffusion, und man erkennt, daß $D$ der Diffusionskoeffizient ist. An diese Entwicklung läßt sich noch eine wichtige Überlegung anknüpfen. Wir haben angenommen, daß die einzelnen Teilchen alle auf dasselbe Koordinatensystem bezogen seien. Dies ist jedoch nicht nötig, da die Bewegungen der einzelnen Teilchen voneinander unabhängig sind. Wir wollen nun die Bewegung jedes Teilchens auf ein Koordinatensystem beziehen, dessen Ursprung mit der Lage des Schwerpunktes des betreffenden Teilchens zur Zeit $t=0$ zusammenfällt, mit dem Unterschiede, daß jetzt $f(x, t) d x$ die Anzahl der Teilchen bedeutet, deren $X$-Koordinaten von der Zeit $t=0$ bis zur Zeit $t=t$ um eine Größe gewachsen ist, welche zwischen $x$ und $x+d x$ liegt. Auch in diesem Falle ändert sich also die Funktion $f$ gemäß Gleichung (1).” [20, p. 558.]

39 See $[19,84]$ 
suspended particles under the influence of gravitation. As a further example, Einstein considered a system involving heat radiation, a charged harmonic oscillator in thermal equilibrium with a gas and heat radiation, thus establishing a bridge between thermodynamics, kinetic theory, and electrodynamics.

In further papers, Einstein analyzed voltage fluctuations in a condenser [22], and he returned to the issue of heat radiation [24]. Following up on work by Smoluchowski, he also dealt with the phenomenon of critical opalescence [25], showing that critical opalescence and the blue color of the sky, while not obviously related to each other, are both due to density fluctuations caused by the molecular constitution of matter. Einstein determined the pressure fluctuations in black-body radiation from the condition that the momentum they convey to a small mirror moving through the radiation precisely compensates for the momentum lost due to the average radiation pressure on the mirror. ${ }^{40}$ The application of techniques developed for Brownian motion to the problem of heat radiation supported Einstein's controversial claim that Planck's formula for the energy spectrum of heat radiation is not compatible with the classical understanding of radiation. ${ }^{41}$ His results show that the fluctuations due to the radiation field can neither be exclusively explained by interference phenomena of classical radiation nor by statistical fluctuations in a gas of light quanta that is conceived as a collection of classical particles.

While Einstein's work on Brownian motion had repercussions in a broad variety of fields, its central impact was, as pointed out in the beginning, on the acceptance of atomism in the early twentieth century. This impact was made possible above all by the pathbreaking experiments of Jean Perrin, that were publicized in numerous articles and books, and in particular in his best-selling and very readable "Les Atomes," published in 1913 [41]. Perrin began his experiments in 1908 and pursued them very much in line with Einstein's thinking on the subject. He then tested a formula equivalent to Einstein's for the vertical distribution of suspended particles under the influence of gravitation. ${ }^{42}$ Perrin had also realized that the analogy established by van't Hoff between an ideal gas and a solution could be extended to colloidal solutions and suspensions, and that this analogy provides an excellent means for obtaining evidence in favor of the atomistic hypothesis. ${ }^{43}$

Most striking was, however, Perrin's detailed, quantitative confirmation of almost all of Einstein's predictions for the stochastic behavior of suspended particles, thus transforming the latter's "invention" of Brownian motion into powerful experimental evidence for the atomistic hypothesis. Earlier experiments were either merely qualitative in nature, e.g., Felix Ehrenhaft's observations of displacements of aerosol particles, Victor Henri's cinematographical measurements of displacements of suspended particles, ${ }^{44}$ or Max Seddig's study of the temperature dependence of Brownian motion; ${ }^{45}$ or they were quantitative in character, e.g., those by Svedberg [53] but still conceived within the conceptual framework of the kinetic theory, assuming that one could actually measure the velocity of Brownian particles. ${ }^{46}$ In 1907 Einstein even wrote a paper dedicated to correcting basic flaws in Svedberg's work [23]. ${ }^{47}$

Perrin, on the other hand, was fully aware that the work of Einstein and Smoluchowski had established a new conceptual basis for the analysis of Brownian motion. In his masterpiece "Les Atomes" he wrote [42]:

Einstein and Smoluchowski have defined the activity of the Brownian motion in the same way. Previously we have been obliged to determine the "mean velocity of agitation" by following as

\footnotetext{
${ }^{40}$ A similar argument is given in more detail in [29].

${ }^{41}$ See [7].

42 Although Einstein's name is mentioned in [39] in connection with the validity of the equipartition theorem for suspended particles, none of his papers are cited.

43 This conceptual background is discussed at length in [40, pp. 166ff.].

${ }^{44}$ See [15] and [32]; for a discussion of Henri's work, see [36, p. 126].

45 See $[47,48]$. For contemporary discussions of Seddig's work, see the discussion remarks by Einstein and Seddig in [28], and [40, p. 204]; for a recent account, see [36, pp. 125-126].

46 See [52, pp. 856-859].

${ }^{47}$ For Svedberg's attempt to defend his experimental analysis, see [54]. For a review of criticisms of Svedberg's work, see [33, pp. 210-212].
} 
nearly as possible the path of a grain. Values so obtained were always a few microns per second for grains of the order of a micron. But such evaluations of the activity are absolutely wrong.

In 1909, Einstein gratefully wrote to Perrin:

I would have thought it impossible to investigate Brownian motion with such precision; it is fortunate for this material that you have taken it up. ${ }^{48}$

Ironically, the confirmation of the age-old atomistic hypothesis came in a historical moment when the massive evidence accumulated in its favor hinted at the limits of the classical understanding of atomism as well. This eventually gave way to an understanding of matter on the basis of a new physics just underway - not least due to Einstein's contributions.

\section{Looking back at a revolution}

Einstein's exploration of the statistical properties of physical processes, such as Brownian motion, amounted to a reversal of perspective with respect to that of classical physics, as he was well aware himself. In his papers on statistical physics, this becomes particularly clear with regard to his interpretation of Boltzmann's principle, which relates the thermodynamic entropy of a physical system to the statistical probability of its states. In a lecture on Boltzmann's principle from $1910,{ }^{49}$ Einstein argued that this principle can be applied in two different ways. When starting from a complete atomistic picture of a system, one can calculate the probability of its states and then determine, with the help of Boltzmann's principle, the entropy and hence the thermodynamic behavior of the system. This is the perspective of classical kinetic theory.

For Einstein, however, the real significance of Boltzmann's principle was rather its reverse application to the case for which no complete atomistic picture of a system was available, as was the case for those systems that were suspected to exhibit non-classical behavior such as heat radiation. In that case, the most important application of Boltzmann's principle was, in Einstein's view, to infer from the observed thermodynamic behavior of a system the statistical probability of its single states. One would thus be able to judge the extent to which the system deviates from the behavior expected according to classical thermodynamics, for instance, by exhibiting the kind of fluctuation behavior represented by Brownian motion. This reversal of perspective is another instance of the Copernicus process mentioned above.

The profound conceptual implications of the atomistic revolution triggered by Einstein's work on Brownian motion also become apparent from the discussion in his 1910 lecture of the question of whether physical facts are causally connected in a complete way. He argued that - in view of the unpredictable irregularity of Brownian motion - this question has to be definitely answered in the negative. Einstein concluded, however, that the very fact that we are able to obtain the statistical laws for such fluctuation phenomena suggests that, on a theoretical level, we have nevertheless to maintain the presupposition of a complete causal determination of physical events, although we can never hope to receive an immediate confirmation of this conception by ever more refined observations - a remarkable stance at the dawn of quantum mechanics.

\section{References}

[1] AEA: Albert Einstein Archives, The Jewish National and University Library, The Hebrew University of Jerusalem, Israel, http://www.alberteinstein.info.

[2] L. Boltzmann, Vorlesungen über Gastheorie, Part 1, Theorie der Gase mit einatomigen Molekülen, deren Dimensionen gegen die mittlere Weglänge verschwinden (Johann Ambrosius Barth, Leipzig, 1896).

48 Einstein to Jean Perrin, 11 November 1909, [11, Doc. 186]. “Ich hätte es für unmöglich gehalten, die Brown'sche Bewegung so präzis zu untersuchen; es ist ein Glück für diese Materie, dass Sie sich ihrer angenommen haben."

49 “Über das Boltzmann'sche Prinzip und einige unmittelbar aus demselben fliessende Folgerungen.” Vorlesung für die Physikalische Gesellschaft Zürich, 2 November 1910, Zangger Nachlaß, Zentralbibliothek Zürich. 
[3] L. Boltzmann, "Vorschlag zur Restlegung gewisser physikalischer Ausdrücke", in: Verhandlungen der Gesellschaft Deutscher Naturforscher und Ärzte, 70. Versammlung zu Düsseldorf, 19-24 September 1898, pp. 67-68; reprinted in: Wissenschaftliche Abhandlungen, edited by Fritz Hasenöhrl (Vogel, Leipzig, 1909).

[4] R. Brown, Edinb. New Philos. J. 5, 358-371 (1828).

[5] S. G. Brush, Arch. Hist. Exact Sci. 5, 1-36 (1968).

[6] S. G. Brush, The Kind of Motion We Call Heat: A History of the Kinetic Theory of Gases in the 19th Century, Book 1, Physics and the Atomists. Book 2, Statistical Physics and Irreversible Processes (North-Holland, Amsterdam, 1976).

[7] J. Büttner, J. Renn, and M. Schemmel, Stud. Hist. Philos. Mod. Phys. 34, 37-59 (2003).

[8] A. Cotton and H. Mouton, Les ultramicroscopes et les objets ultramicroscopiques (Masson, Paris, 1906).

[9] CPAE 1, edited by J. Stachel, D. C. Cassidy, R. Schulmann, and J. Renn, The Collected Papers of Albert Einstein, Vol. 1, The Early Years, 1879-1902 (Princeton University Press, Princeton, 1987).

[10] CPAE 2, edited by J. Stachel, D. C. Cassidy, J. Renn, and R. Schulmann, The Collected Papers of Albert Einstein, Vol.2, The Swiss Years: Writings, 1900-1909 (Princeton University Press, Princeton, 1989).

[11] CPAE 5, edited by M. J. Klein, A. J. Kox, and R. Schulmann, The Collected Papers of Albert Einstein, Vol. 5, The Swiss Years: Correspondence, 1902-1914 (Princeton University Press, Princeton, 1993).

[12] CPAE 9, edited by D. Kormos Buchwald, R. Schulmann, J. Illy, D. J. Kennefick, and T. Sauer, The Collected Papers of Albert Einstein, Vol.9, The Berlin Years: Correspondence, January 1919-April 1920 (Princeton University Press, Princeton, 2004).

[13] G.L. De Haas-Lorentz, Die Brownsche Bewegung und einige verwandte Erscheinungen (Friedrich Vieweg und Sohn, Braunschweig, 1913).

[14] R.J. Deltete, The Energetics Controversy in Late Nineteenth-Century Germany: Helm, Ostwald and Their Critics, 2 vols., Ph.D. dissertation, Yale University (1983).

[15] F. Ehrenhaft, Sitz.ber., Kaiserl. Akad. Wiss. Math.-Nat.wiss. Kl. IIa 116 1139-1149 (1907).

[16] A. Einstein, Ann. Phys. (Leipzig) 4, 513-523 (1901), reprinted in this volume (pp. 88-98).

[17] A. Einstein, Ann. Phys. (Leipzig) 8, 798-814 (1902), reprinted in this volume (pp. 100-116).

[18] A. Einstein, Ann. Phys. (Leipzig) 9, 417-433 (1902), reprinted in this volume (pp. 118-134).

[19] A. Einstein, Ann. Phys. (Leipzig) 14, 354-362 (1904), reprinted in this volume (pp. 155-163).

[20] A. Einstein, Ann. Phys. (Leipzig) 17, 549-560 (1905), reprinted in this volume (pp. 182-193).

[21] A. Einstein, Ann. Phys. (Leipzig) 19, 371-381 (1906), reprinted in this volume (pp. 248-258).

[22] A. Einstein, Ann. Phys. (Leipzig) 22, 569-572 (1907), reprinted in this volume (pp. 292-295).

[23] A. Einstein, Zeitschrift für Elektrochemie und angewandte physikalische Chemie 13, 41-42 (1907), reprinted in [10, Doc. 40].

[24] A. Einstein, Physikalische Zeitschrift 10, 323-324 (1909).

[25] A. Einstein, Ann. Phys. (Leipzig) 33, 1275-1298 (1910), reprinted in this volume (pp. 368-391).

[26] A. Einstein, "Theoretische Atomistik", in: Die Kultur der Gegenwart. Ihre Entwicklung und ihre Ziele, Part 3 , Sect. 3, Vol. 1, Physik, edited by P. Hinneberg and E. Warburg (B. G. Teubner, Leipzig, 1915), pp. 251-263.

[27] A. Einstein, Autobiographical Notes: A Centennial Edition, translated and edited by P.A. Schilpp (La Salle, Illinois, 1979).

[28] A. Einstein et al., Phys. Z. 10, 779-780 (1909), reprinted in CPAE 2, Doc. 58.

[29] A. Einstein and L. Hopf, Ann. Phys. (Leipzig) 33, 1105-1115 (1910), reprinted in this volume (pp. 357-367).

[30] F. M. Exner, Ann. Phys. (Leipzig) 2, 843-847 (1900).

[31] A. Fick, Ann. Phys. Chem. 4, 59-86 (1855).

[32] V. Henri, C.R. Acad. Sci. 146, 1024-1026 (1908).

[33] M. Kerker, ISIS 67, 190-216 (1976).

[34] K. von Nägeli, Sitz.ber., Kgl. Bayerische Akad. Wiss. München, Math.-phys. Kl. 9, 389-453 (1879).

[35] W. Nernst, Z. Phys. Chem. Stöchiometrie Verwandtschaftslehre 2, 613-637 (1888)).

[36] M. J. Nye, Molecular Reality: A Perspective on the Scientific Work of Jean Perrin (Macdonald, London, 1972)

[37] W. Ostwald, Lehrbuch der allgemeinen Chemie, 2nd rev.ed., Vol.1, Stöchiometrie (Wilhelm Engelmann, Leipzig, 1891).

[38] W. Ostwald, Lehrbuch der allgemeinen Chemie. 2nd rev. ed. Vol. 2, part 1, Chemische Energie (Wilhelm Engelmann, Leipzig, 1893).

[39] J. Perrin, C.R. Acad. Sci. 146, 967-970 (1908). 
[40] J. Perrin, "Les preuves de la réalité moléculaire (Etude spéciale des émulsions)", in: La théorie du rayonnement et les quanta. Rapports et discussions de la réunion tenue à Bruxelles, du 30 octobre au 3 novembre 1911. Sous les auspices de M.E. Solvay, edited by P. Langevin and M. de Broglie, (Gauthier-Villars, Paris).

[41] J. Perrin, Les Atomes (Félix Alcan, Paris, 1913).

[42] J. Perrin, Atoms, translated by D. Ll. Hammick (Ox Bow Press, Woodbridge, Conn., 1990).

[43] H. Poincaré, La science et l'hypothèse (E. Flammarion, Paris, 1902).

[44] J. Renn, "Einstein's Controversy with Drude and the Origin of Statistical Mechanics: A New Glimpse from the 'Love Letters'”, in: Einstein: The Formative Years, 1879-1909, Einstein Studies, Vol. 8, edited by D. Howard and J. Stachel (Birkhäuser, Boston, 2000), pp. 107-157.

[45] J. Renn, Phys. J. 3, 49-55 (2004).

[46] J. Renn and R. Rynasiewicz, “The Puzzle of Einstein's Annus Mirabilis”, in: Cambridge Companion to Einstein, edited by M. Janssen and C. Lehner (Cambridge University Press, New York, in press); J. Renn, "Einstein as a Disciple of Galileo: A comparative study of Concept Development in Physics", in: Einstein in Context, edited by M. Beller, R. Cohen, and J. Renn (Cambridge University Press, Cambridge, 1993).

[47] M. Seddig, Sitz.ber., Ges. Beförderung der gesammten Naturwissenschaften, 182-188 (1907).

[48] M. Seddig, Phys. Z. 9, 465-468 (1908).

[49] H. Siedentopf and R.A. Zsigmondy. Ann. Phys. (Leipzig) 10, 1-39 (1903).

[50] M. von Smoluchowski, Ann. Phys. (Leipzig) 21, 756-780 (1906).

[51] W. Sutherland, Philos. Mag. J. Sci. 44, 493-498 (1897).

[52] T. Svedberg, Z. Elektrochem. angewandte phys. Chem. 12, 853-860 (1906).

[53] T. Svedberg, Z. Elektrochem. angewandte phys. Chem. 12, 909-910 (1906).

[54] T. Svedberg, Z. Phys. Chem. Stöchiometrie Verwandtschaftslehre 71, 571-576 (1910).

[55] J.H. Van 't Hoff, Z. Phys. Chem. Stöchiometrie Verwandtschaftslehre 1, 481-508 (1887).

[56] R.A. Zsigmondy, Zur Erkenntnis der Kolloide. Über irreversible Hydrosole und Ultramikroskopie (Gustav Fischer, Jena, 1905). 